\title{
Kernos
}

Revue internationale et pluridisciplinaire de religion grecque antique

9 | 1996

Varia

\section{Les Charites à Athènes et dans l'île de Cos}

\section{Vinciane Pirenne-Delforge}

URL : http://journals.openedition.org/kernos/1167

DOI : $10.4000 /$ kernos. 1167

ISSN : 2034-7871

\section{Éditeur}

Centre international d'étude de la religion grecque antique

\section{Édition imprimée}

Date de publication : 1 janvier 1996

ISSN : 0776-3824

\section{Référence électronique}

Vinciane Pirenne-Delforge, «Les Charites à Athènes et dans l'île de Cos », Kernos [En ligne], 9 | 1996, mis en ligne le 21 avril 2011, consulté le 21 avril 2019. URL : http://journals.openedition.org/ kernos/1167; DOI : 10.4000/kernos. 1167 


\section{Les Charites à Athènes et dans l'île de Cos}

Leur nom évoque la grâce en ses multiples manifestations : la grâce de l'apparence, la beauté; la grâce du geste et de l'attitude, le talent et la noblesse; la grâce conférée par l'exploit, la gloire'; la gratitude, la faveur accordée ou rendue. Ainsi, les Charites étaient associées à un idéal esthétique et éthique qui caractérisa très tôt la vie de la polis grecque ${ }^{2}$. Néanmoins, il serait abusif de réduire les dévotions qui leur étaient rendues dans les cités à la seule manifestation d'un tel idéal. Les Charites n'étaient pas de simples abstractions personnifiées et divinisées ${ }^{3}$. Il est tout aussi hasardeux d'en faire des divinités aux prérogatives naturalistes dont le cours du temps aurait élargi les domaines d'intervention aux artifices de l'esprit humain et de la vie en sociétét. L'origine des différents cultes des Charites est, comme souvent, largement inconnaissable. La tradition voulait que trois pierres tombées du ciel à Orchomène aient depuis la plus haute antiquité été vénérées sous le nom des trois Grâces. Les mythes locaux autour de ce culte structurent une réalité complexe qui intègre tout autant la fertilité et la prospérité du territoire que la finesse politique d'Étéocle, le roi ancestral qui fonda le culte et fit du sacrifice aux Charites un mode d'exercice du pouvoir ${ }^{5}$. Comment dès lors, si ce n'est en vertu d'une hypothèse prétendument fondatrice, dissocier l'ordre naturel et biologique des réalités socio-politiques quand il surgissent sous nos yeux intimement liés? Délaissant les scénarios fondateurs et originels, quelles qu'en soient les

1 Sur la charts agonistique, voir B. MACLACHLAN, The Age of Grace. Charis in Early Greek Poetry, Princeton Univ. Press, 1993, p. 87-123.

2 Voir à cet égard la réflexion stimulante de Chr. MEIER, La politique et la grâce. Antbropologie politique de la beauté grecque, Paris, Seuil, 1987 [or. all. Berlin, 1985].

3 À l'instar d'autres «notions » du même type, comme Éros ou Peitho. $C f$. V. PIRENNEDetforge, Le culte de la Persuasion. Peitbo en Grèce anctenne, in RHR, 208 (1991), p. 395-413. - Sur la question des personnifications, voir l'intéressante introduction de H.A. SHAPIRO, Personifications in Greek Art. The Representation of Abstract Concept 600-400 B.C., Zürich, 1993 (Akantbus Crescens, 1), p. 12-29. Ni Charis ni les Charites n'apparaissent parmi les entrées qui organisent l'ouvrage.

4 L.R. FARNELL (The Cults of the Greek States, V, Oxford, 1909, p. 427-431) developpa la théorie of the transformation of elemental into spiritual powers. - Les réserves d'A. BRELICH sur ces prétendues « déesses de la végétation » sont éloquentes (Paides e Parthenoi, Roma, 1969, p. 222, n. 33).

5 PAUS., IX, 34, 6 - 38, 1; ÉPHORE, 70 F 152 Jacoby (= Schol. BT Homère, Il., IX, 381); STRABON, IX, 40 (C414-415). Cf. M. RoCCHI, Contributi allo studio delle Cbarites (I), in StudClas, 18 (1979), p. 10-15; A. SCHACHTER, Cults of Boeotia. 1. Acbeloos to Hera, London, 1981 (BICS Suppl, 38, 1), p. 140-144. 
réussites, on s'attachera plus particulièrement aux Charites athéniennes et à celles de l'île de $\operatorname{Cos}^{6}$, après un bref détour par les textes archaïques d'Homère et d'Hésiode, qui offrent un bon point de départ à la réflexion.

Dès l'Iliade, Charis apparaît dans la famille olympienne. C'est comme épouse d'Héphaïstos qu'elle accueille Thétis venue commander de nouvelles armes pour son fils ${ }^{7}$. Le dieu forgeron n'oublie pas qu'il doit la vie à la bienveillance de la Néréide qui le sauva des eaux ${ }^{8}$. Forger une panoplie guerrière pour Achille sera le paiement de sa dette. Charis s'est substituée à l'Aphrodite infidèle de l'Odyssée pour signifier cette reconnaissance. Quelques chants auparavant, Hypnos, convaincu par Héra de neutraliser Zeus dans un doux sommeil, demandait pour prix de son intervention la main de Pasithéa, « la plus jeune des Charites $»$. Héra concluait le marché en affirmant que la reconnaissance survivrait longtemps à la transaction. La Charite épousée en serait une sorte de mémorial. Dans l'Odyssée, ce sont les Charites - un pluriel indifférencié - qui baigneront Aphrodite venue se réfugier à Paphos de Chypre après la révélation de son adultère ${ }^{10}$. L'épopée homérique connaît donc la tension entre le singulier et le pluriel pour évoquer les Charites : Charis, d'une part, Pasithéa, de l'autre, dont le statut de cadette appelle à l'existence une ou plusieurs soeurs aînées. Liées au don et à la reconnaissance ${ }^{11}$, elles ont en outre l'âge d'être épousées, l'âge de la séduction.

La Théogonie d'Hésiode est pareillement significative, sous d'autres formes. Après les grandes crises cosmogoniques, lordre de Zeus est en place : les Titans rebelles sont relégués au fond du Tartare et Typhon est vaincu. Surviennent alors les unions successives de Zeus : Métis d'abord, qui lui permet d'asseoir son autorité sur l'intelligence rusée; Thémis ensuite, qui lui donne les Heures et les Moires; Eurynomé, Océanide, à la beauté tant aimée, qui met au monde trois Charites aux belles joues. Naîtront encore Perséphone de Déméter, les neuf Muses de Mnémosyne, Apollon et Artémis de Léto, etc. Ce qui nous intéresse en l'occurrence, c'est l'apparition de ces trois triades de divinités féminines, dont les personnalités sont individualisées : les Heures sont Eunomia, Diké, Eiréné, les Moires, Clotho, Lachésis, Atropos, et les Cbarites, Aglaié,

\footnotetext{
6 Certains cultes des Charites ont étê récemment étudiés avec beaucoup de finesse par Maria ROCCHI en deux articles successifs sur Orchomène et Sparte, d'une part, Athènes, Paros, Thasos, et l'Arcadie, d'autre part (Contributo allo studio delle Charites I \& II, in StudClas, 18 [1979], p. 5-16; 19 [1980], p. 19-28). Il me semble possible d'approfondir sa réflexion sur Athènes et intéressant de lui adjoindre l'exemple de Cos.

7 HOM., Il, XVIII, 382-392.

8 HOM., $h$., XVIII, 406-407.

9 HOM., Il., XIV, 263-276.

10 HOM., Od., VIII, 360-366. Cf. aussi Hymne ps.-bom. Aphrodite, 58-66.

11 Sur le thème du don dans l'antiquité et sa postérité, on verra D. VIDAL, Les gestes du don. $A$ propos des "Trois Grâces", in M.A.U.S.S., Ce que donner veut dire. Don et intérêt, Paris, La Découverte, 1993, p. 60-77. Je dois cette intéressante référence à l'amabilité d'Alain Ballabriga.
} 
Euphrosyne et Thalie ${ }^{12}$. À ce stade de l'ordonnancement du monde, le temps est venu de régler l'existence des humains sous l'autorité de Zeus. C'est alors qu'apparaissent les divinités collectives féminines dont on trouve bien davantage trace dans les cultes que ces larges groupes primordiaux, féminins eux aussi, que sont les Océanides ou les Néréides ${ }^{13}$. Les Heures, les Moires et les Charites permettent de déterminer les cadres de l'existence humaine: les Moires en limitent le cours, les Heures créent les conditions de la prospérité (bon ordre, justice, paix) au fil des saisons, les Charites offrent les composantes de la vie en société : Thalie, dont le nom évoque à la fois l'abondance et les fêtes qui la saluent, Euphrosyne la bonne disposition d'humeur, Aglaié le resplendissement. La dernière, la plus jeune, est femme d'Héphaïstos dans la Théogonie ${ }^{14}$, ce qui permet de voir dans son nom, notamment, une référence aux arts que patronne le forgeron divin, desquels l'éclat et la charis illuminent la couronne de Pandore ${ }^{15}$. De surcroît, au cours de sa création, Pandore se verra offrir par les Heures des guirlandes de fleurs et par les Charites des colliers, produits significatifs de leurs prérogatives respectives ${ }^{16}$.

Un des leitmotive artistiques de la représentation des Charites - tant littéraire qu'iconographique - est leur familiarité avec la danse. Elles dansent sur l'Olympe, en compagnie d'Artémis, d'Aphrodite, des Heures, d'Hébé et d'Harmonie; elles ont l'âge de ces korai humaines qui forment des chœurs en l'honneur des dieux ${ }^{17}$. Sur le mode de la danse, elles apparaissent aussi aux côtés de Dionysos ${ }^{18}$. Dans le fameux hymne clétique chanté par les Seize Femmes d'Élis en l'honneur du dieu du vin, c'est en compagnie des Charites qu'il est invité à venir « d'un pied de taureau » dans son temple ${ }^{19}$.

\footnotetext{
12 HÉs., Thëog., 901-911.

13 Si les Néréides peuvent être honorées comme telles, les Océanides ne le sont qu'à titre individuel.

14 HÉs., Théog., 945-946.

15 HÉs., Théog., 583.

16 HÉs., Trav. G Jours, 73-75.

17 E.g. Hymne ps.-bom. à Apollon, 194-196; PIND., Ol., XIV, 10-13. Sur l'agora d'Élis, PAUSANIAS (VI, 24, 6-7) décrit les trois statues acrolithes des Charites : l'une porte une rose, la deuxième une branche de myrte, la troisième un astragale, l'attribut qui signifie la jeunesse. - Sur l'importance de la danse dans la religion grecque, $c f$. S.H. LONSDALE, Dance and Ritual Play in Greek Religion, Baltimore, 1993.

18 ARISTOPH., Gren., 324-336.

19 PlUT., Questions grecques, $36(=$ Mor, 299a-b). Cf. Chr. G. BROWN, Dionysus and the Women of Elis, in GRBS, 23 (1982), p. 305-314; V. MITSOPOULOS-LEON, Zur Verebrung des Dionysos

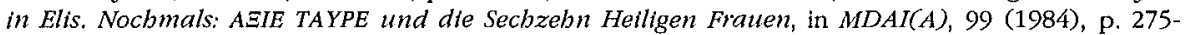
290. - Les Charites et Dionysos possèdent un autel en commun dans l'Altis d'Olympie : PAUS., V, 14,
} 10. 
Dès les années 480-460, les Charites faisaient partie des divinités honorées avant les Eleusinia et recevaient également le sacrifice d'une chèvre lors des Mystères d'Éleusis ${ }^{20}$. De tels sacrifices, apparemment réservés aux Kerykes, l'une des deux grandes familles sacerdotales d'Éleusis, laissent entendre qu'il existait au moins un autel des Charites à Éleusis, au plus un sanctuaire et un culte régulier à Athènes $^{21}$. Dans un autre registre, Aristophane, quand il fait jurer Trygée en faveur de la Paix, les unit à Hermès, aux Heures, à Aphrodite, à Pothos contre Arès et Enyalios ${ }^{22}$, tandis que les femmes célébrant les Thesmophories, dans la pièce qui porte leur nom, prient Ploutos, Kalligeneia, Kourotrophos, Hermès et les Charites ${ }^{23}$. Elles accompagnent également la danse de Iacchos dans la parodie des mystères du même Aristophane ${ }^{24}$. Les déesses apparaissent aussi dans la tragédie athénienne ${ }^{25}$ et l'Hippolyte d'Euripide les associe aux mariages ${ }^{26}$. Ce sont néanmoins des textes tardifs qui permettent de jeter davantage de lumière sur les Charites athéniennes.

\section{Pausanias et les Charites}

Visitant Athènes au IIe siècle de notre ère, Pausanias contourne les flancs de la citadelle, termine le parcours du peripatos sous le bastion d'Athéna Niké et se dirige vers les Propylées de Mnésiclès. Il énumère d'abord les peintures qui ornent la pinacothèque de l'aile gauche; ensuite ${ }^{27}$

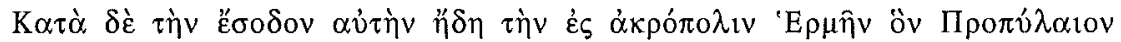

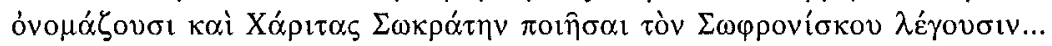

À l'entrée même de l'Acropole se trouvent dès lors l'Hermès que l'on surnomme Propylaios et les Charites, œuvres, dit-on, de Socrate, le fils de Sophronisque...

Pausanias n'en dira pas davantage sur le bastion. Ce n'est qu'aux livres III et V qu'il reviendra incidemment sur la statue de l'Athéna Victoire que les Athéniens

$20 I G, \mathrm{I}^{3}, 5(=L S C G, 4)$. Cf. aussi $L S C G, \mathrm{n}^{\circ} 10,1.81-83$ (datée entre 403 et 399 av, J.-C.). $C f$. K. CLINTON, IG $I^{2} 5$, the Eleusinia, and the Eleusinians, in $A J P b, 100$ (1979), p. 1-12, surtout p. 7-8.

21 Un calendrier sacrificiel athénien de la première moitié du ve siècle intègre une restitution - trop importante pour l'ériger en certitude - où les Charites réapparaissent : $I G, \mathrm{I}^{2}, 840,1.13-14$ $(=L S C G, 1),[\mathrm{X} \alpha \rho \rho] \_\sigma ı$.

22 ARISTOPH., Paix, 456-457. Cf. aussi Oiseaux, 1320-1321.

23 ARISTOPH., Thesm., 295-300. Cf. aussi les vers 120-122.

24 Cf. supra n. 18.

25 Eur., Bacch., 414; Héraclès, 673; Hélène, 1341. Dans le Cyclope, les Charites sont prises à témoin de l'attachement de Polyphème pour les jeunes garçons (581-584).

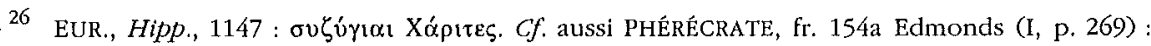

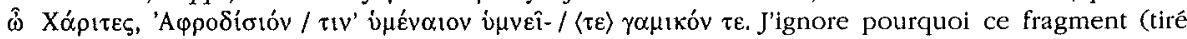
du Lexicon Sabbatticum, 2, 22) n'apparaît dans aucune autre édition, antérieure ou ultérieure.

27 PAUS., I, 22, 8 (trad. J. Pouilloux, C.U.F.). 
ont voulue sans ailes pour la garder toujours de leur côté ${ }^{28}$. Au livre II, il évoquera, à propos de l'Hécate d'Égine, une statue trimorphe de la déesse qualifiée d'Epipyrgidia et sise, à Athènes, près du temple de l'Athéna Aptère ${ }^{29}$. Quant aux Charites, il faut attendre le livre IX et les développements historicolégendaires sur l'Orchomène de Béotie pour obtenir des compléments d'information.

Selon un procédé d'exposition qui lui est cher, Pausanias présente les traditions locales d'une cité avant d'en proposer un parcours descriptif. Orchomène n'échappe pas à la règle et les temps anciens de sa splendeur évanouie sont ressuscités par la vertu mythique des séquences généalogiques. D'après les Béotiens, le roi Étéocle - deuxième de la lignée après son père Andreus - fut le premier homme à sacrifier aux Charites. Elles étaient trois, mais le souvenir du nom qu'il donna à chacune s'est perdu ${ }^{30}$. Pausanias ouvre alors une longue digression sur les déesses, fournissant bon nombre d'indications qui, lorsqu'il ne s'agit pas des renseignements déjà distillés au fil de ses visites, offrent de précieux compléments laissés dans l'ombre lors de la présentation des lieux.

En fait, les Lacédémoniens, eux, affirment que les Charites sont deux et que Lacédémon, fils de Taygète, les établit et leur donna les noms de Klêta et Phaënna. De tels noms conviennent bien pour des Charites, de même que ceux qu'elles portent parmi les Athéniens. En effet, les Athéniens eux aussi honorent depuis longtemps (éк $\pi \alpha \lambda \alpha 10 \hat{v}$ ) les Charites Auxo et Hégémoné. Car le nom de Karpo n'est pas celui d'une Charite mais d'une Heure. $\check{A}$ la seconde Heure, les Athéniens accordent des honneurs en compagnie de Pandrose, en appelant la

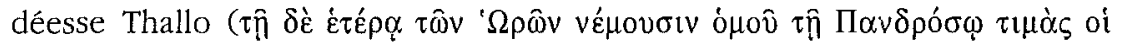

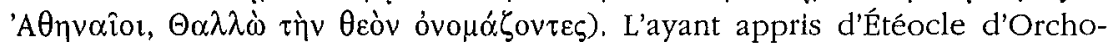
mène, on a aujourd'hui coutume de prier trois Charites. Ainsi, l'Apollon sculpté pour les Déliens par Angelion et Tektaios porte trois Charites dans la main. À Athènes devant l'entrée de l'Acropole, les Charites sont trois également et ils célèbrent auprès d'elles une initiation secrète pour la plupart des gens ( $\pi \alpha \rho \dot{\alpha} \delta \grave{\varepsilon}$

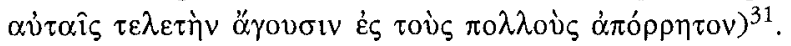

Suit alors une série de références littéraires dûment identifiées, Pamphos, le premier à avoir chanté les Charites, Homère, Hésiode, avec qui Onomacrite s'entend pour les nommer Aglaié, Euphrosyne et Thalie, Antimachos, qui en fait les filles d'Aiglé et du Soleil, Hermésianax qui compte Peitho parmi elles ${ }^{32}$. Puis

28 PAUS., III, 15, 7 (par comparaison avec l'Enyalios enchaîné de Sparte); V, 26, 6 (Calamis aurait imité la statue athénienne en sculptant une Niké sans ailes).

29 PAUS., II, 30, 2.

30 PAUS, IX, 35, 1.

31 PAUS., $\mathrm{X}, 35,1-3$.

32 PAUS., IX, 35, 4-5. 
vient le registre iconographique. Pausanias déclare ignorer qui, le premier, a dénudé les Grâces alors que sculpteurs et peintres du temps passé les drapaient. Et pour clore l'inventaire des représentations les plus notables des Charites habillées, Pausanias revient un instant à Athènes ${ }^{33}$ : « Socrate, le fils de Sophronisque, a réalisé les agalmata des Charites devant l'entrée de l'Acropole d'Athènes, " La digression est terminée. Pausanias reprend imperturbablement le fil de la généalogie royale d'Orchomène.

Pour Sparte, le Périégète répète une information livrée en bonne place au livre $\mathrm{III}^{34}$. Pour Athènes, il avait déjà attribué les Charites de l'entrée de l'Acropole au philosophe Socrate, mais le tableau se complique en se précisant. Depuis les temps anciens ( $\dot{\kappa} \kappa \pi \alpha \lambda \alpha 10 \hat{)}$ ), les Athéniens honorent deux Charites, dont les noms apparaissent pour la première fois dans la Périégèse, Auxo et Hégémoné. D'où Pausanias a-t-il tiré son information ? D'une présentation synthétique sur les Grâces dans un ouvrage général sur les dieux ? D'un lä̈us en forme d'excursus sur les célèbres déesses dans une histoire d'Orchomène ${ }^{35}$ ? Quelle que soit sa source, elle devait présenter une erreur aux yeux de Pausanias - ou d'une autre source qu'il utilisait conjointement (trois Charites athéniennes : Auxo, Hégémoné, Karpo). En effet, il entame sans préavis une sorte de polémique érudite, rendant Karpo au groupe des Heures dont on l'avait manifestement soustraite. Quant à Pandrose, je ne pense pas que Pausanias l'ait comptée au nombre des Heures athéniennes ${ }^{36}$. Il précise simplement qu'elle partageait certains honneurs avec la seconde des Heures ( $\tau \hat{\imath} \delta \dot{\varepsilon} \dot{\varepsilon} \tau \varepsilon \rho \alpha$ ), qu'ils appelaient Thallo. Deux Heures, Karpo et Thallo, deux Charites, Auxo et Hégémoné, telle aurait été la configuration ancienne de ces groupes féminins à Athènes ${ }^{37}$. Que penser de l'influence d'Étéocle dans le passage du deux au trois? A. Schachter a bien montré que le groupement en triade était récurrent pour les divinités féminines en Béotie ${ }^{38}$. En outre, c'est depuis la Béotie qu'Hésiode a canonisé les triades féminines. Pindare, le poète béotien, chantera les Charites d'Orchomène en les baptisant, à l'instar

33 PAUS., IX, 35, 6-7.

34 PAUS., III, 18, 6. Sur les cultes des Charites à Sparte, cf. ROCCHI, art. cit. (n. 6), I, p. 15-16.

35 C'est l'hypothèse de C. ROBERT (cité par A. LESKY, art. Thallo, in RE, V A 1 [1934], c. 1214) qui faisait de Kallipos de Corinthe, historien d'Orchomène cité par le seul Pausanias (IX, 29, 2; 38, 10), la source des chapitres IX, 34, 6-38. F. JACOBY en doute (IIIb Kommentar [1955], p. 180, à propos de $385 \mathrm{~F}$ 1-2) et l'on doit convenir de la difficulté de ce type de Quellenforschung.

36 Ainsi que le comprend P. BRULÉ, La fille d'Atbènes. La religion des flles à Athènes à l'époque classique. Mythes, cultes et sociêté, Paris, 1987, p. 34.

37 De la même manière, les Cécropides semblent bien avoir été deux et puis trois : P. BRULÉ, Aritbmologie et polythétsme, in Les grandes figures religieuses. Fonctionnement pratique et symbolique dans l'Antiquité (Besançon, 25-26 avril 1984), Paris, 1986 (Annales litt. de l'Univ. de Besançon, 329. Centre de Recherches d'Histolie Ancienne, 68. Lire les polytbéismes, 1), p. 35-47.

38 A. SCHACHTER, Some Underlying Cult Patterns In Boeotia, in Telresias, Suppl. 1 (1972), p. 17-18. Cf. V. PIRENNE-DELFORGE, L'Apbrodite grecque, Liège-Athènes, 1994 (Kernos Suppl., 4), p. $279-280$. 
d'Hésiode, Aglaíe, Euphrosyne et Thalie ${ }^{39}$. Le poids de cette tradition a dû contribuer à uniformiser la représentation triple de ces rondes de Nymphes,

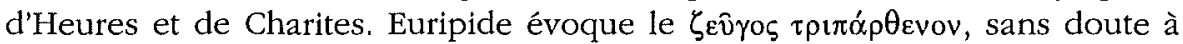
propos des Cécropides ou des Érechthéides, tandis que Sophocle parle des

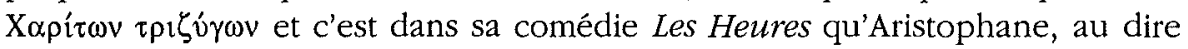
d'Hésychios, emprunte abusivement l'image d'un attelage pour désigner trois esclaves $^{40}$. C'est très clair sur un plan iconographique. Xénophon ne faisait-il pas dire à Socrate combien ces danses réjouissaient la vue, même s'il était impossible d'identifier les gracieuses jeunes filles représentées ${ }^{41}$ ?

\section{Les Charites et les jeunes gens d'Athènes}

À suivre Pausanias, les Heures et les Charites athéniennes étaient donc originellement des couples, Karpo et Thallo pour les Heures, Auxo et Hégémoné pour les Charites. Les Heures offrent par leurs noms l'image de leurs prérogatives : protectrices de la croissance de la végétation, on sait par Philochore que les Athéniens les priaient pour contrer les chaleurs excessives, faire venir la pluie en temps opportun, et qu'elles aidaient Dionysos à faire mûrir le raisin ${ }^{42}$. Quant à Auxo et Hégémoné, elles sont respectivement « celle qui fait pousser, qui fait croître » et «celle qui guide ». Leur sphère d'intervention est différente, car si l'on interprète Auxo à la lumière d'Hégémoné, il ne s'agit plus de favoriser la croissance des plantes, mais bien celle de ceux que l'on guide ${ }^{43}$. Or, précisément, le serment des éphèbes athéniens en appelle au témoignage, parmi d'autres divinités, de Thallo, d'Auxo et d'Hégémoné, de même qu'aux bornes de la patrie, aux blés, aux orges, aux vignes, aux olives et aux figues ${ }^{44}$.

\footnotetext{
39 PIND., Ol., XIV.

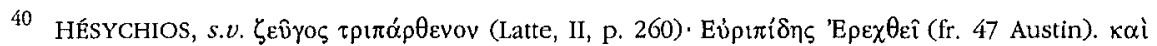

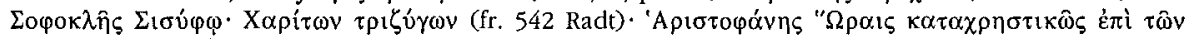

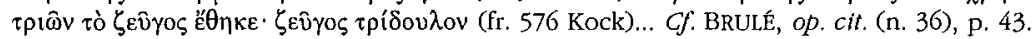

41 XÉN, Banquet, 7, 5. Cf. Th. HADZISTELIOU PRICE, Double and Multiple Representations in Greek Art and Religious Thought, in JHS, 91 (1971), p. 48-69; E. MITROPOULOU, Charites-Nymphs, in Pulpudeva, 5 (1986), p. 83-97. - Un relief du Musée du Cinquantenaire à Bruxelles (inv. A 1150), de provenance sans doute laconienne, représente de manière assez fruste trois figures féminines de profil, portant chacune un attribut (collier, fruit, fleur). La pierre porte l'inscription Kópac $\Sigma \omega \tau$ tó, $\zeta$, datable du début du VIe ou du début du Ve siècle av. J.-C. Une fernme a donc dédié ces trois kópaı, qui évoquent irrésistiblement les Charites, les Heures, les Moires ou même les Érinyes, dans un beau cas d'indétermination qui donne matière à réflexion. Le relief est repris par V. MAKAIRA dans l'art. Horai du $L I M C, \mathrm{~V}(1990)$, p. 502-506, $\mathrm{n}^{\circ}$ 8. - Sur le caractère courotrophe de ces groupes féminins d'un point de vue littéraire, $c f$. J. WILKINS, The Young of Atbens: Religion and Society in Herakleidai of Eurtpides, in CQ, 40 (1990), p. 329-339.

42 PHILOCHORE, 328 F $173 \mathrm{~J}$. (= ATHÉNÉE, XIV, 656a); $328 \mathrm{~F}$ 5b (= ATHÉnÉE, II, 38c). Cf. MACHAIRA, art. cit. (n. 41).

43 Cf. ROCCHI, art. cit. (n. 6), II, p. 19-20.

44 L. ROBERT, Études épigraphiques et philologiques, Paris, 1938, p. 296-307; M. BOCK, Die Scbuurgötter der Epbeben von Acharnai, in Wiener Jabreshefte, 33 (1941), p. 46-55; C. PÉLÉKIDIS, 
Le serment est ancien, on l'a maintes fois affirmé ${ }^{45}$. Outre les Olympiens comme Zeus, Hestia, Arès, ou d'autres personnalités de facture ancienne comme Aglauros et Enyalios, les éphèbes athéniens invoquent des déesses protectrices de leur croissance et donc garantes du renouvellement de la population. Elles assurent également les moyens de subsistance de la cité qui sont eux aussi appelés en garants du serment. On constate clairement dans ces témoignages, outre leur dimension guerrière, la solidarité des notions de fertilité et de fécondité, agraire et humaine, nécessaires à l'existence même de la cité. Grâce au témoignage de Pausanias, on peut affirmer que le nom de ces protectrices a servi à singulariser les Heures et les Charites. Il est néanmoins impossible d'en identifier clairement le processus : la Thallo du serment fut-elle toujours une des Heures ? Auxo et Hégémoné étaient-elles originellement des Charites? Autant de questions insolubles car Pausanias est le seul à déterminer les composantes de ces groupes dont le nom est générique dans la plupart de nos autres sources sur Athènes ${ }^{46}$.

Ainsi en va-t-il du sacrifice appelé $\gamma \alpha \mu \eta \lambda \hat{i} \alpha$. Surtout attestée chez les orateurs attiques qui désignent de la sorte le repas offert dans sa phratrie par un jeune marié présentant son épouse ${ }^{47}$, la gamèlia a piqué la curiosité des lexicographes qui ont accumulé à son sujet bon nombre de contradictions. Synonyme de kovpê̂tıs ou sacrifice des éphèbes sur le point de se marier pour l'un ${ }^{48}$, sacrifice lors de la présentation des filles dans la phratrie pour l'autre ${ }^{49}$ ou, conformément aux dires des orateurs, banquet nuptial offert aux phratères ${ }^{50}$, seul l'Etymologicon Magnum précise que le sacrifice était destiné à Héra, à

Histolre de l'éphébie attique, Paris, 1962, p. 113, 217; G. DAUX, Le serment des épbèbes atbéniens, in REG, 84 (1971), p. 370-383; P. SIEWERT, The Ephebic Oath in the Fifth Century Athens, in JHS, 97 (1977), p. 102-111; BRULÉ, op. cit. (n. 36), p. 33-35.

45 Sur les antécédents initiatiques de l'éphébie, cf. P. VIDAL-NAQUET, Le chasseur noir et l'origine de l'épbébie atbénienne, in J.-P. VERNANT, P. VIDAL-NAQUET, La Grèce ancienne 3. Rites de passage et transgressions, Paris, 1992, p. 119-147 lédition originale de 1968, revue dans Le Cbasseur noir; Paris, $1991^{3}$, p. 141-175].

46 À l'exception d'une scholie aux Nuées d'Aristophane qui les nomme Peitho, Aglaïa et Thalie (773a Holwerda [Scholia in Aristophanem, IV. Jo. Tzetzae Commentarii in Aristophanem, II. Commentarium in Nubes, Groningen, 1960, p. 560]). Cf. aussi la Souda, s.v. Xর́ $\rho 1 \tau \alpha \varsigma$ (Adler, IV,

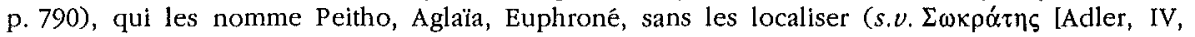
p. 403], les Charites apparaissent, mais ne sont pas individualisées).

47 E.g. ISÉE, III, 76; 79; VII, 18; 20. Cf. P. SCHMITT, Athéna Apatouria et la ceinture : les aspects féminins des Apatourtes à Atbènes, in Annales(ESC), 32 (1977), p. 1059-1073.

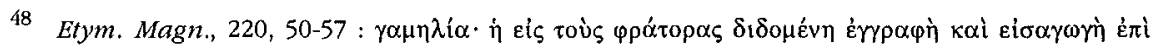

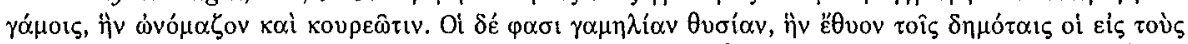

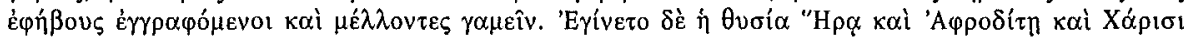

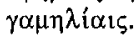

49 POLluX, VIII, 107. Cf. J. LABARBE, L'âge correspondant all sacrifice du xov́petov et les données bistoriques du sixième discours d'Isée, in BAB, 39 (1953), p. 358-394, surtout p. 364-365, et BRULE, op. cit. (n. 36), p. 402-403.

50 Harpocration, s. $v$. $\gamma \alpha \mu \eta \lambda \hat{i} \alpha$ (Dindorf, I, p. 77); HÉsychios, s. $v . \gamma \alpha \mu \eta \lambda \hat{i} \alpha$ (Latte, I, p. 361). 
Aphrodite et aux Charites qualifiées de Gamèliai ${ }^{51}$. Le rôle d'Héra comme protectrice du mariage à Athènes est trop connu pour qu'il soit nécessaire d'y insister ${ }^{52}$. Quant à Aphrodite, une découverte récente a confirmé sa présence dans un tel cadre puisque, au titre d'Ourania, elle recevait une drachme pour le sacrifice préliminaire au mariage en pleine époque classique ${ }^{53}$. Les Charites nuptiales interviennent donc, à Athènes, en relation avec l'échange par excellence que constitue le mariage, à un moment mal déterminé, car la notice lexicographique qui les évoque juxtapose diverses notations ${ }^{54}$. Ainsi, la mention des éphèbes sur le point de se marier est étonnante, car les Athéniens dans cette situation n'étaient plus des «éphèbes " 55 . S'il est donc possible de voir, à la suite de Pollux, dans la gamèlia un sacrifice offert dans les phratries, d'un côté, par les pères qui engagent leur fille toute jeune, de l'autre, par le mari qui $\mathrm{y}$ introduit son épouse ${ }^{56}$, c'est moins évident pour les garçons. La confusion pourrait trouver son origine dans le patronage accordé par les Charites aux éphèbes, d'une part, et leur protection du mariage, d'autre part.

Les Charites athéniennes sont donc concernées par des moments importants de la vie en société : la croissance des jeunes, le mariage qui implique, à terme, la succession des générations et l'équilibre du corps civique ${ }^{57}$.

\section{Les cultes du bastion et de l'agora}

C'est à l'entrée des Propylées que Pausanias a vu l'Hermès Enagônios et les Charites. Le texte n'est pas suffisamment explicite pour préciser ce que le visiteur eut sous les yeux. S'agit-il d'un groupe d'Hermès et des Charites réalisé par Socrate? Seules les Charites lui sont attribuées par les auteurs anciens ${ }^{58}$ et, dès la fin du XIXe siècle, des reliefs néo-attiques représentant trois femmes, dont l'un fut trouvé sur l'Acropole, ont été interprétés comme des transpo-

\footnotetext{
51 Etym. Magnum, 220, 50-57.

52 P. LÉVÊQUE, L. SÉCHAN, Les grandes divinités de la Grèce, Paris, 1966, p. 180-183.

53 L'inscription stoichedon, datée du début du IVe siècle av. J.-C., se trouve sur un thesauros en

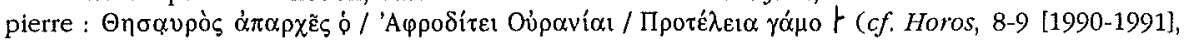
p. 17-44; $S E G, \mathrm{XLI}, 182$ ). Cette découverte confirme l'interprétation que j'avais donnée du culte d'Aphrodite Ourania dans ma thèse (L'Aphrodite grecque, op. cit. [n. 38], p. 15-25). Cf. PAUS., II, 34, 12, pour un sacrifice préliminaire au mariage à Hermione.

54 Cf. supra, n. 40.

55 Sur l'âge des jeunes gens au mariage, $c f$. J.H. OAKLEY, R.H. SINOS, The Wedding in Ancient Athens, Univ. of Wisconsin Press, 1994, p. 10.

56 BRULÉ, op. cit. (n. 36), p. 402-403.

57 M. ROCCHI (art. cit. [n. 6], I, p. 15-16) a bien montré qu'à Sparte, les cultes des Charites devaient avoir aussi une relation étroite avec les jeunes au moment de leur entrée dans la communauté des citoyens.

58 DIOGÈNE LAËrCE, II, 19; Schol. ARISTOPH., Nuées, 773a (cf, supra, n, 46); Souda, s.v.

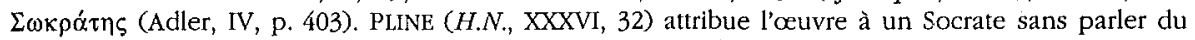
philosophe. Cf. E. SCHWARZENBERG, Die Grazien, Bonn, 1966, p. 16-19.
} 
sitions archaïsantes des «Charites de Socrate » ${ }^{59}$. Au livre I, Pausanias ne qualifie pas l'œuvre dont il parle. C'est par le nom des dieux représentés qu'il l'identifie. Au livre IX, les Charites de Socrate sont sous sa plume des agalmata, appellation que notre auteur réserve dans la grande majorité des cas à des statues divines en ronde-bosse ${ }^{60}$. Le style des reliefs en question laisse penser qu'ils furent élaborés en fonction de statues (agalmata) classiques imitant le style sévère, et non à partir d'un relief de ce type ${ }^{61}$. L'hypothèse des trois agalmata est encore appuyée par deux reliefs mutilés du IVe siècle trouvés sur l'Acropole. Les fragments représentent une Athéna identifiée avec beaucoup de vraisemblance comme l'Athéna Niké du Bastion avec, en arrière-plan, trois petites figures féminines hiératiques portant chacune un polos majestueux, en qui l'on a reconnu la triade des Charites ${ }^{62}$. Leur ressemblance avec les korai de l'Acropole est frappante.

Un ultime indice de la localisation des Charites à l'entour du bastion de Niké est l'existence d'une prêtrise conjointe d'Artémis Epipyrgidia et des Charites, attestée par deux inscriptions récentes (Ier s. av. et Ile s. ap. J.-C.) qui l'associent à la charge de pyrphoros des Mystères d'Éleusis ${ }^{63}$. Cette Artémis est l'Hécate Epipyrgidia connue par le texte de Pausanias qui la situe sur le bastion et indique qu'Alcamène fut le premier à sculpter la déesse sous une triple forme ${ }^{64}$. D'autre part, un $\pi \alpha v \alpha \gamma \eta ́ s$ (un officiel lié lui aussi aux Mystères, mais dont la fonction est indéterminée ${ }^{65}$ ) est également prêtre d'Hermès $\Pi v \lambda \eta n ̃ \eta \varsigma$

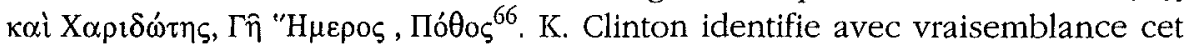

59 E.B. HARRISON, art. Charites, in LIMC, III (1986), p. 201-202; O. PALAGIA, A New Relief of the Graces and the Charites of Socrates, in Sacris Erudirl, 31 (1989-1990), p. 347-356 (= Opes Atticae. Miscellanea philologica et bistorica $R$. Bogaert et $H$. Van Looy) : spéc. n. 42.

60 Cf. mon étude sur le vocabulaire religieux de Pausanias, en préparation. C'est aussi le mot

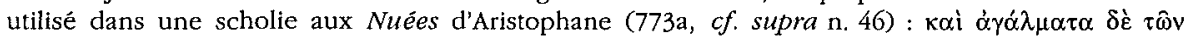

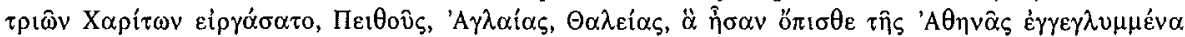

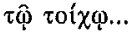

61 HARRISON, art. cit. (n. 59), p. 201-202, choisissant la première des deux hypothèses de B.S. RIDGWAY, The Severe Style in Greek Sculpture, Princeton, 1970, p. 114-121.

62 O. WALTER, Bescbreibung der Reliefs im kleinen Akropolismuseum in Atben, Wien, 1923, $\mathrm{n}^{\circ}$ 274-275; HARRISON, art. cit. (n. 59), $\mathrm{n}^{\circ}$ 11-12. Le mérite de cette identification revient à L. BESCHI, Contributi di topografia ateniese, in $A S A A, 45-46$ (1967-1968), p. 534-536. Le relief $\mathrm{n}^{\circ} 276$ de WALTER (= HARRISON $n^{\circ} 13$ ) pose davantage de problèmes d'interprétation. - Un relief archaïsant d'une collection particulière en Belgique offre un parallèle bien mis en lumière par O. PALAGIA, art. cit. (n. 59).

63 P. ROUSSEL, Un nouveau document concemant le genos des Kńpvкes, in AIPbO, 2 (1934), p. 819-834 (= Mélanges Bidez); K. CLINTON, The Sacred Officials of the Eleusintan Mysteries, in TAPbS, 64, 3 (1974), p. 50-52, 1. 10-11, et p. 94; $I G, \mathrm{II}^{2}, 5050$.

64 Cf. supra, n. 29. À l'époque hellénistique s'est développé à Athènes le type iconographique de l'Hékataion entouré de trois Charites dansant. Il est tentant de voir là une influence de la proximité de la triple Hécate et des trois Charites de l'entrée de l'Acropole. Cf. HARRISON, art. cit. (n. 59), $\mathrm{n}^{\circ}$ 28-34; H. SARIAN, art. Hekate, LIMC, VI (1992), n²17-233.

65 CLINTON, op. cit. (n. 63), p. 95-96.

$66 I G, \mathrm{II}^{2}, 3664$. $G f$. CLINTON, ibid. 
Hermès « de la porte » avec l'Hermès Propylaios évoqué par Pausanias, et voit dans la seconde épiclèse une trace de sa relation avec les Charites ${ }^{67}$.

Où se situaient ces statues? Pausanias parle de l'entrée de l'Acropole chaque fois qu'il cite les Charites athéniennes. La localisation la plus naturelle serait dès lors à l'intérieur des Propylées de Mnésiclès, du côté du bastion, ce qu'invite encore à choisir l'épiclèse Propylaios qui qualifie l'Hermès tout proche $^{68}$. Une indication du même auteur rend néanmoins cette interprétation difficile. Car s'il y eut vraiment une télétè « secrète pour la plupart des gens » célébrée auprès des Charites, les Proypylées ne sont guère un endroit adéquat $^{69}$. Nous sommes donc ramenés vers le bastion lui-même.

Pour étudier l'évolution de la topographie des lieux, on dispose d'une excellente étude sur l'Athéna Niké du bastion à l'entrée de l'Acropole, qui a récemment permis de préciser les idées jusque-là assez confuses ${ }^{70}$. Les premiers indices d'un culte de la déesse en ces lieux - un autel inscrit et un reposoir pour une statue - remontent à la période 580-560, ce qui correspond aux grands travaux entrepris sur l'Acropole et à une période de renouveau cultuel assez généralisé à Athènes ${ }^{71}$. L'étude attentive de ce que l'on a longtemps pris pour une eschara a montré qu'il s'agissait en fait du reposoir de la statue de culte qui devait être en pierre et assise ${ }^{72}$. Par Pausanias, on sait que la statue de Niké était sans ailes ${ }^{73}$. D'après Héliodore d'Athènes, Périégète lui-aussi, elle portait une grenade dans une main et un casque de l'autre ${ }^{74}$. Ira Mark a montré de façon convaincante que cette statue, contrairement au sanctuaire proprement dit, avait survécu aux destructions qui ont marqué l'acropole au Ve siècle $^{75}$. Le bastion semble être resté en ruines jusqu'au début des années 440 ,

67 Ibid.

68 Cf. J. TRAvlos, Pictorial Dictionary of Ancient Atbens, London, 1971, p. 150, fig, 200.

69 Une remarque déjà formulée par S, GSELL, art. Gratiae, in DAREMBERG-SAGLIO-POTTIER, Dict. des Ant., II, 2 (1896), p. 1664, n. 9. Remarque reprise par BESCHI, art. cit. (n. 62), p. 536. - Cette télétè n'est pas autrement connue. Pausanias a sans doute puisé cette information à la source qui lui donnait l'identité des Charites. Selon que les auteurs adoptent une interprétation «agraire » ou "initiation juvénile », la télétè a servi leur propos (cf. PALAGIA, art. cit. [n. 59], p. 352; RoCCHI, art. cit. [n. 6], II, p. 23). S'il y eut jamais une cérémonie secrète en l'honneur des Charites athéniennes, on serait tenté d'y voir un parallèle avec le rituel athénien de l'arrhéphorie, peu connu de la plupart des gens au dire de Pausanias (I, 27,3). Le Périégète ne qualifie pas la cérémonie de télétè, mais le secret entoure l'ensemble des actions menées par les petites filles entre le plateau et le flanc nord de l'Acropole ( $c f$. PIRENNE-DELFORGE, op. cit. [n. 38], p. 498-59).

70 I. MARK, The Sanctuary of Atbena Nike in Atbens. Arcbitectural Stages and Cbronology, Princeton, 1993 (Hesperia Suppl, 26).

71 H.A. SHAPIRO, Att and Cult under the Tyrants in Athens, Mainz, 1989, p. 1-5; 21-27; MARK, op. cit. (n. 71), p. 34-36.

72 MARK, op. cit. (n. 71), p. 20-30.

73 Cf. supra, n. 28.

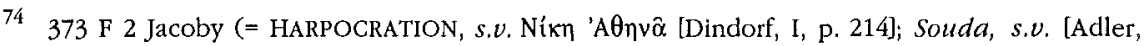
III, p. 466])

75 MARK, op. cit. (n. 71), p. 123-125. 
moment où sa reconstruction provisoire a été décidée, sous la forme d'une simple cella en forme de $\Pi^{76}$. Quant à l'état que nous connaissons aujourd'hui, il aurait été achevé vers les années $420-418^{77}$. La nature de la divinité honorée, l'Athéna Victoire, au vu des attributs de sa statue, grenade et casque, mêle à des intérêts guerriers des préoccupations d'un autre ordre. La grenade, et plus généralement ces fruits ronds désignés du nom générique de $\mu \hat{\eta} \lambda \alpha$ sont les marques iconographiques de la sphère «fertilité-fécondité ». On trouve trace de cet arrière-plan dans l'Ion d'Euripide, dont la représentation date de la période d'achèvement de la dernière phase du sanctuaire ${ }^{78}$. D'une part, le chœur invoque Athéna Niké pour qu'elle ne laisse pas la maison royale d'Athènes sans descendance et, au moment de la reconnaissance entre Créuse et son fils, la reine prend Athéna Niké à témoin de son serment sur la paternité d'Apollon ${ }^{79}$.

Le bastion connut donc, à la fin du Ve siècle, une réorganisation profonde et l'installation d'une construction prestigieuse. C'est de la même époque que datent les statues des Charites attribuées à Socrate et l'Hécate(-Artémis) Epipyrgidia d'Alcamène. Ces œuvres relèvent manifestement du même souci de revalorisation du lieu que l'érection du temple d'Athéna Niké. L'Hermès, qu'il soit de Socrate ou même d'Alcamène, comme certains l'ont pensé ${ }^{80}$, date vraisemblablement de la même époque. Le raisonnement par association topographique ne peut tenir lieu de seule démonstration, mais la convergence dans la datation de ces représentations iconographiques laisse penser que leur regroupement n'est pas fortuit, à une époque où tout cet espace était à organiser.

Quant à savoir si ces divinités étaient, comme Athéna, déjà honorées à cet endroit au VIe siècle, le problème est délicat. La seule certitude repose sur leurs qualités respectives. L'Hécate du bastion remplit une fonction de gardienne des portes que lui connaît déjà Eschyle ${ }^{81}$, mais les qualités de courotrophe d'Artémis-Hécate sont largement répandues ${ }^{82}$. Athéna Niké intègre une double dimension de protection de la cité, symbolisée par la dualité de ses attributs : casque et fruit du cru. Les Charites, enfin, interviennent dans le serment éphé-

76 Ibid., p. 36-68.

77 Ibid., p. 69-92.

78 Ibid., p. 77-92.

79 EUR., Ion, 452-471, 1528-1531. - À Cos, où nous retrouverons bientôt les Charites, Niké recevait, au Ile $s$. av. J.-C., des sacrifices lorsque des mariages étaient célébrés dans son sanctuaire (LSCG, $\left.\mathrm{n}^{\circ} 163,1.1-2\right)$.

80 E.g. D. MUSTI-L. BESCHI, Pausania. Guida della Grecia. Libro I: L'Attica, Napoli, $1987^{2}$ [1982], p. 343-344. Contra G. SIEBERT, art. Hermes, in LIMC, V (1990), n 42-46, et comm. p. 374.

81 Fr. 388 Radt (= Schol. THÉOCRITE, Id., II, 35/36a). Cf. S.I. JOHNSTON, Hekate Soteira, Scholars Press, 1990 (American Classical Studies, 21), p. 21-28; SARIAN, art. cit. (n. 64).

82 Sur la localisation précise - tout hypothétique - de la statue d'Alcamène sur le bastion, $c f$. en dernier lieu M.D. FULLERTON, The Location and Archatsm of the Hekate Eptpyrgidia, in $A A$ (1986), p. 669-675. 
bique, un bel exemple de la dimension complexe de la protection du territoire, qui mêle les évocations militaires aux réalités agraires. Un indice ténu de l'ancienneté possible des Charites et d'Hécate tient au type archaïque que leurs statues ont épousé en cette fin de Ve siècle ${ }^{83}$.

Un relief bien connu de la fin du VIe siècle et conservé au Musée de l'Acropole (inv. 702) montre un aulète de profil menant trois femmes de face, dansant main dans la main. La dernière tient un garçon nu et de plus petite taille. On y a vu une scène de culte banale ${ }^{84}$, des Nymphes, les Charites ${ }^{85}$ ou encore les Cécropodies menées par Hermès et tenant le petit Erichthonios par la main ${ }^{86}$. La relation entre les Charites et les éphèbes permettrait de voir dans le garçon nu, non pas un enfant, mais bien un éphèbe, reconnaissable par sa nudité et dont la petite taille signifie la condition humaine aux côtés des divinités. Dans cette hypothèse, le culte serait antérieur à l'œuvre de Socrate et peut-être déjà situé sur le bastion, auprès d'Athéna Nikér ${ }^{87}$.

En 229 avant notre ère était établi à l'angle nord-est de l'agora athénienne un téménos du Démos et des Charites ${ }^{88}$. En cette même fin de IIIe siècle, le

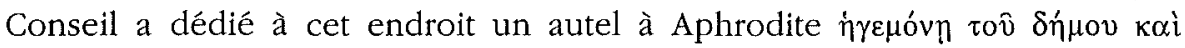
Xópı $\iota^{89}$. Or c'est notamment en présence du prêtre du Démos et des Charites que les éphèbes sacrifiaient sur le foyer public du Prytanée à leur entrée en charge ${ }^{90}$. La fonction est récente, mais le Démos et les Charites de l'agora assurent sans doute une forme de continuité avec le patronage que les déesses, dont une Hégémoné, accordent depuis longtemps aux éphèbes de la cité. L'autel dédié par la Boulé permet de le penser, de même que deux décrets récemment mis au jour à Rhamnonte. Il s'agit, pour l'un, d'un décret de soldats athéniens, en l'occurrence les éphèbes en garnison à Aphidna et à

\footnotetext{
83

Cf. PAlAGia, art. cit. (n. 59), p. 354-355.

84 H. PAYNE, G.M. YOUNG, Arcbaic Marble Sculpture from the Acropolis, London, 1936, p. 4950.

85 H. LECHAT, Hermès et les Charites, in BCH, 13 (1889), p. 467-479.

86 BRULÉ, op. cit. (n. 36), p. 45.

87 Avant l'érection des Propylées de Mnésiclès, l'espace est suffisant pour compter divers cultes. Après la construction, on pourrait placer le sanctuaire des Charites dans l'espace laissé libre entre le sanctuaire de Niké, les Propylées de Mnésiclès et le mur de soutènement qui s'élève jusqu'au portique d'Artémis Brauronia sur le plateau. Cf. BESCHI, art. cit. (n. 62), p. 536; PALAGIA, art. cit. (n. 59), p. 354-355 et fig. 17. - Une triade dansant sur la frise du temple d'Athéna Niké pourrait représenter les Charites : E.G. PEMBERTON, The East and West Friezes of the Temple of Athena Nike, in AJA, 76 (1972), p. 303-310, surtout p. 309; HARRISON, art. cit. (n. 59), $\mathrm{n}^{\circ} 22$.

88 R. WYCHERLEY, The Athenian Agora, III. Literary and Epigraphical Testimonia, Princeton, 1957, p. 59-61; J.H. OLIVER, Demokratla, the Gods, and the Free World, New York, 1968, p. 106-107.

$89 I G, \mathrm{II}^{2}, 2798$. Cf. PIRENNE-DELFORGE, op. cit. (n. 38), p. 39-40 pour la bibliographie concernant cette fondation.

90 PÉlÉKIDIS, op. cit. (n. 44), p. 217.
} 
Rhamnonte, en l'honneur du général Nicomachos, daté de l'année 222/1 avant J.-C. ${ }^{91}$ Il a été placé à Aphidna et à Rhamnonte dans le biéron d'Aphrodite Hégémoné, déesse à laquelle le stratège Nicomaque a sacrifié à sa sortie de charge. L'autre décret, daté de 220 , est très mutilé, mais la stèle semble avoir été placée elle aussi dans le sanctuaire d'Aphrodite.

Ces documents nous mènent à l'époque hellénistique et il est délicat de projeter les informations qu'ils nous donnent sur les siècles antérieurs. Tout au plus fera-t-on l'hypothèse qu'Aphrodite, à une époque indéterminée, a accordé son crédit de divinité olympienne à la Charite Hégémoné en charge des éphèbes à date ancienne. La présence de la déesse en un point stratégique de la défense athénienne n'est pas fondée sur des qualités guerrières dont j'ai montré ailleurs la faible part ${ }^{92}$. Je pense que c'est la qualité d'éphèbes des soldats en garnison qui explique l'apparition d'Aphrodite Hégémoné.

\section{Les Charites de $\operatorname{Cos}^{93}$}

Un très beau relief datant des alentours de l'an 400 avant notre ère a été trouvé en 1966 à Mesaria, à quelque $6 \mathrm{~km}$ à l'ouest de la ville moderne de Cos. Il représente trois jeunes femmes engagées main dans la main dans un pas de danse derrière un autel. Devant elles, un homme barbu de petite taille lève la main droite en signe d'hommage. La tête du dieu Pan est représentée dans une sorte d'encadrement rocheux dans l'angle supérieur gauche du relief, au-dessus

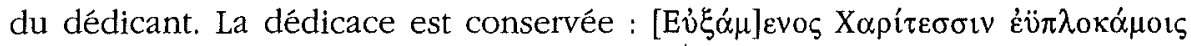

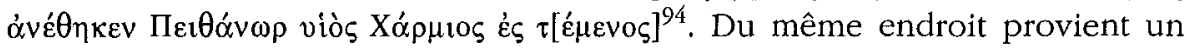

91 J'ai déjà évoqué ce décret dans ma thèse (PIRENNE-DELFORGE, op. cit. [n. 38], p. 39-40). Le texte est aujourd'hui disponible : $S E G, \mathrm{XLI}, 90$, auquel on peut ajouter le $\mathrm{n}^{\circ} 91$.

92 PIRENNE-DELFORGE, op. cit. (n. 38), p. 450-454. - Sur les relations entre le caractère courotrophe de certaines divinités et l'univers de la guerre, $c f$. R. LONIS, Guerre et religion en Grèce à l'époque classique, Paris, 1979, passim. - À Sparte, les Charites sont notamment honorées près du dromos, le lieu d'exercice des jeunes gens, à côté du sanctuaire des Dioscures, d'rlithye, d'Apollon Karneios et d'Artémis Hégémoné (PAUS., III, 14, 6).

93 Le culte des Charites est bien attesté dans les îles de la mer Égée. À Thasos, des inscriptions du début du Ve siècle av. J.-C., sous des reliefs ( $I G$, XII 8, 358), signalent que les Charites ne pouvaient recevoir ni chèvre ni porc en sacrifice. Pour les Nymphes et Apollon Nymphagète, on ne sacrifiait ni mouton ni porc, et l'on ne pouvait pas entonner le péan. - D'après, entre autres, CALlimaQUE (Aitia, fr. 3-5 Pfeiffer) et le Ps.-APOLLODORE (II, 15, 7), le sacrifice parien aux Charites se déroulait sans flûtes ni couronnes depuis que Minos avait appris la mort d'Androgée à l'occasion d'une telle cérémonie ( $c f$. ROCCHI, art. cit. [n. 6], II, p. 25-26, pour tout le dossler). SCHWARZENBERG (op. cit. [n. 58], p. 4-7) y voyait la célébration de la végétation qui meurt et renaît, ROCCHI (ibid.) interprète l'absence de couronnes comme un retour à une époque antérieure à Prométhée et y voit un rite de capo d'anno. Je serais plutôt tentée, compte tenu du caractère courotrophe des déesses, de rapprocher ces interdits des prescriptions qui frappent la célébration des Hyakinthies à Sparte et de voir dans la mort d'Androgée la référence à une initiation juvénile (sur la fête spartiate, cf. M. PETTERSSON, Cults of Apollo at Sparta, Stockholm, 1992, p. 9-41).

94 HARRISON, art. cit. (n. 59), $\mathrm{n}^{\circ} 24$. À sa bibliographie, il convient d'ajouter : J. BOUSQUET, Le sanctuaire des Charites à Cos, in ZPE, 7 (1971), p. 279; G. KONSTANTINOPOULOS, Avó $\gamma \lambda v \varphi o \mu \varepsilon \tau t \zeta$

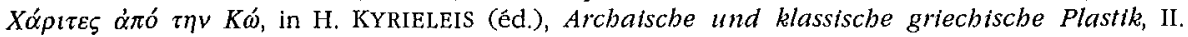


autre relief, d'époque hellénistique (IIe ou Ier siècle), lui aussi dédié aux

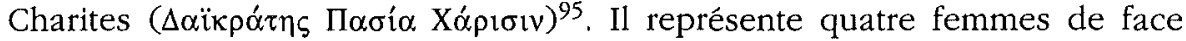
vêtues d'un chiton et d'un himation, portant chacune un polos. La deuxième appuie son avant-bras sur l'épaule de la première au-dessus de laquelle apparaît la tête de Pan. La première et la troisième font un geste de dévoilement. Devant la deuxième et la troisième femme est sculptée une sorte de petite grotte où est couché un personnage de taille réduite, drapé à moitié ${ }^{96}$.

Le premier de ces deux reliefs atteste l'existence d'un culte des Charites antérieur au synœecisme qui intervint à $\operatorname{Cos}$ en 366 avant notre ère ${ }^{97}$. Une réorganisation générale de la vie religieuse dut avoir lieu peu après. Un calendrier cultuel de cette époque ${ }^{98}$ présentait la distribution des honneurs rendus aux dieux, mois après mois ${ }^{99}$. Son caractère fragmentaire ne permet plus d'en connaître qu'une faible part. Un des quatre fragments ne se rapporte à aucun des trois autres et le nom du mois a disparu dans une lacune ${ }^{100}$. La vingtaine de lignes conservées décrit une série de sacrifices accompagnés de prescriptions touchant à la consommation des victimes et aux émoluments des prêtres (1. 1$5,17-21$ ). De la fin de la cinquième ligne au début de la dix-septième, des cérémonies particulières s'adressent aux Charites et à Asia. J'en reproduis le texte d'après F. Sokolowski ( $L S C G, \mathrm{n}^{\circ} 151 \mathrm{D}$ ), mais sans restituer les lacunes des lignes 12,14 et 15 , qui déterminent l'interprétation du texte :

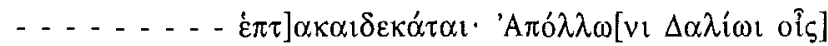

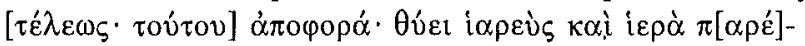

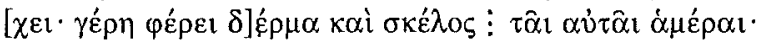

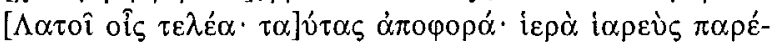

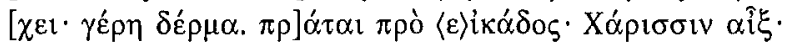

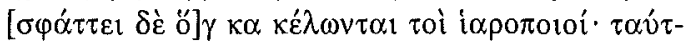

Klassische griechiscbe Plastik. Akten des intern. Kolloquiums vom 22.-25. April 1985 in Atben, Mainz, 1986, p. 135-141.

95 HARRISON, art. cit. (n. 59), n 42.

96 Cf. infra, n. 117.

97 Cf. S.M. SHERWIN-WHITE, Ancient Cos. An bistorical study from the Dorian settlement to the imperial period, Göttingen, 1978, p. 40, 43-72. - Le site ne semble pas encore avoir été fouillé de manière sytématique.

98 Moitié du IVe siècle d'après l'inventeur de la pierre R. HERZOG (Heilige Gesetze von Kos, in Abbandlungen der Preussiscben Akademie der Wissenschaften, Jabrgang 1928, PbilosophiscbHistorische Klasse, Berlin, 1929, p. 7) et SOKOLOWSKI (LSCG, p. 252), dernier quart de ce siècle d'après SHERWIN-WHITE (op. cit. [n. 97], p. 292-293). Cf. B. LE GUEN-POLLET, La vie religieuse dans le monde grec du Ve au IIle siècle avant notre ère. Cbolx de documents épigrapbiques traduits et commentés, Toulouse, 1991, p. 187-190 (= LSCG, $\mathrm{n}^{\circ} 151 \mathrm{~B}=$ SEGRE, op. cit. [n. 112], $\mathrm{n}^{\circ}$ ED 241); J. SVENBRO, Le partage sacrificiel selon une lot sacrée de Cos (IVe siècle avant J.-C.), in Antbropozoologica, premier $\mathrm{n}^{\circ}$ spécial (1987), p. 71-76 ( $\left.=L S C G, \mathrm{n}^{\circ} 151 \mathrm{~A}\right)$.

99 LSCG, $\mathrm{n}^{\circ} 151 \mathrm{~A}-\mathrm{D}$. Les pierres avaient été trouvées et publiées par R. HERzoG, Hellige Gesetze von Kos, in Abbandlungen der Preussiscben Akademie der Wissenscbaften, Jabrgang 1928, Philosophisch-Historische Klasse, Berlin, 1929, $\mathrm{n}^{\circ} 1-4$.

100 LSCG, $\mathrm{n}^{\circ} 151 \mathrm{D}$. 


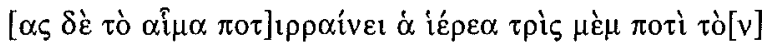

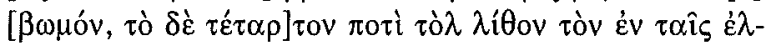

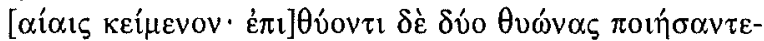

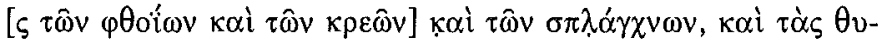

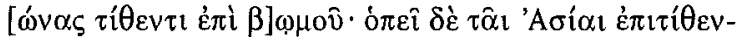

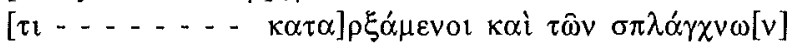

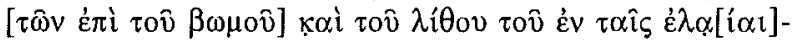

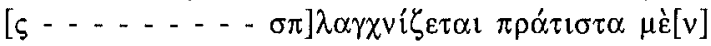

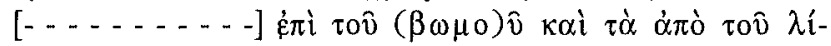

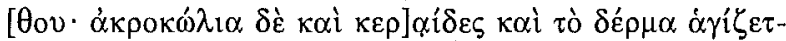

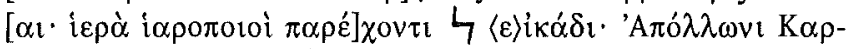

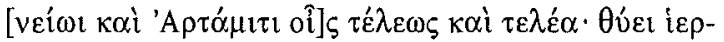

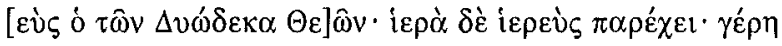
20

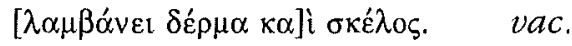

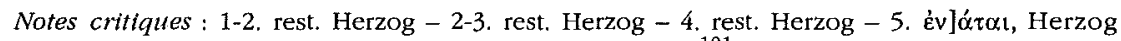

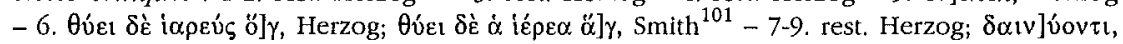

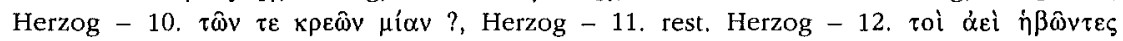

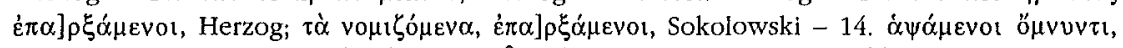

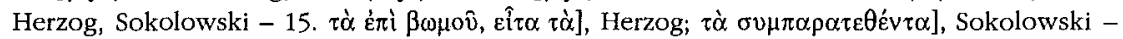

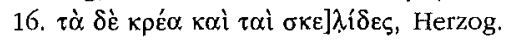

- - - le dix-sept, pour l'Apollon [de Délos un mouton adulte]; il est permis d'en emporter des parts; c'est le prêtre qui sacrifie et qui les fournit consacrées; [comme honoraires, il reçoit] la peau et une patte. Le même jour, pour [Léto une brebis adultel; il est permis d'en emporter des parts; le prêtre les fournit consacrées; [comme honoraires, il a la peau]. Le dix-neuf, une chèvre pour les Charites; [procède à l'égorgement] celui que les hiéropes en chargent; [le sang] de cette chèvre, la prêtresse en arrose trois fois [l'autel, la quatrième fois] la pierre [sise] au milieu des oliviers; ils sacrifient en outre deux portions, qu'ils ont confectionnées, [de gâteaux ronds, de viandes] et d'entrailles, et ils [mettent] les portions sur l'autel; là où c'est pour Asia qu'ils déposent [- - - ] après avoir également préparé l'offrande des entrailles [sur l'autel] et la pierre au milieu des oliviers [- - - - ] en tout premier lieu, sont consommées les entrailles [rassemblées sur] (l'autel) et celles qui viennent de la pierre; [les parties saillantes du corps, les parties cornées] et la peau sont vouées au feu; [les hiéropes] fournissent les parts consacrées. Le vingt, pour Apollon Karneios [et pour Artémis des moutons] adultes, mâle et femelle; c'est le prêtre des Douze Dieux qui sacrifie; le prêtre fournit les parts consacrées; comme honoraires, lil reçoit la peaul et une patte $---^{102}$

101 D.R. SMITH, The bieropoioi on Kos, in Numen, 20 (1973), p. 38-47, surtout p. 42-43.

102 Pour l'étude de cette inscription, j'ai pu bénéficier des conseils du Professeur Jules Labarbe, notamment de précieuses suggestions pour cette traduction, qui reste problématique, mais que j'ai souhaité affronter. 
R. Herzog, l'inventeur de la pierre, décelait dans le cérémonial en l'honneur des Charites la procédure du serment des éphèbes, dont les termes les plus évidents ne sont perceptibles que dans la restitution de la ligne 14, reprise par $\mathrm{F}$. Sokolowski. Une telle lecture est tentante, mais le texte réédité par ce dernier ne permet pas de comprendre quand ont été déposées sur la pierre située au milieu des oliviers les entrailles que les participants consomment $(\sigma \pi \lambda \alpha \gamma \chi v i \zeta \varepsilon \tau \alpha 1,1.14))$. La mention de la pierre à la ligne 14 dépend, en effet, de $\alpha \psi \alpha \alpha ́ \alpha \varepsilon v o r$, qui est restitué. La présence des entrailles sur la pierre est davantage expliquée si l'on considère que le koì coordonne l'autel et la pierre, et non les formes participiales $\dot{\pi} \pi \alpha] \rho \xi \alpha ́ \alpha \varepsilon v o r ~(1.12)$ - auquel je préfère $\kappa \alpha \tau \alpha] \rho \xi \dot{\alpha} \mu \varepsilon v{ }^{103}$ - et $\dot{\alpha} \psi \alpha \alpha \mu \varepsilon v o$ (1. 14). Le simple ö $\mu v v v \tau$ serait donc plus satisfaisant.

L'aspersion de sang qui suit la mise à mort de la chèvre est une entrée en matière connue pour les serments. Par contre, la consommation des entrailles, clairement attestée dans ce calendrier, reste un problème controversé dans la reconstitution des procédures juratoires ${ }^{104}$. Elle pourrait n'avoir pas toujours été prohibée $^{105}$. De plus, le serment des éphèbes, dans sa version athénienne, souligne la solidarité des jeunes gens. La consommation des entrailles pourrait avoir le sens d'une sorte de «communion » renforçant encore le poids du serment $^{106}$. Il faut enfin remarquer que ni la prêtresse ni les hiéropes ne reçoivent d'honoraires, puisque ce qui n'est pas consommé est entièrement voué au feu. En dépit de son caractère partiel, une telle destruction de la victime pourrait favoriser l'hypothèse du serment.

Tout le rituel s'articule donc autour de la chèvre sacrifiée en l'honneur des Charites, sous l'autorité des hiéropes et de la prêtresse. Dans un tel contexte,

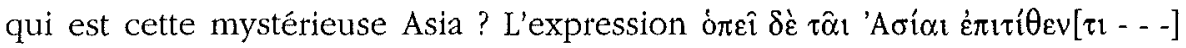
est locative. Or deux lieux en rapport avec le sacrifice apparaissent dans l'inscription : l'autel et la pierre. Il semble peu probable que « là où c'est pour Asia qu'ils déposent... » soit un troisième lieu de performance du rituel. D'après Herzog, Asia devait être une des Charites ${ }^{107}$. F. Sokolowski supposait qu'Asia, une des Océanides d'Hésiode, était honorée en tant que mère des Charites, à la

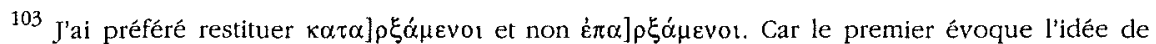
préparation, ce qui permet également de voir apparaître le dépôt des entrailles sur la pierre. Le second évoquerait déjà une aspersion préliminaire quand rien n'indiquerait comment les entrailles sont arrivées là.

104 Cf. W. BURKERT, Greek Religlon, Harvard, 1985, p. 252. Contra J. RuDHARDT, Notions fondamentales et actes constitutifs du culte dans la Grèce classique, Paris, 1992², p. 284.

105 PAUSANIAS $(V, 24,10)$ entrevoit la possibilité que l'animal ait pu être consommé à partir d'une époque qu'il ne précise pas.

106 HERZOG, op. cit. (n. 99), p. 14. - Le serment athénien souligne bien la solidarité du groupe dans la défense du territoire, cf. supra, n. 44.

107 HERzOG, op. cit. (n. 99), p. 13. Avant d'opter pour l'interprétation de l'hapax $\theta 0$ óv $\alpha$ comme "Opferportion ", Herzog avait supposé que Thyoné était un nom propre - connu par ailleurs -, désignant ici l'une des Charites (ibid.). Sa propre hypothèse ne l'a pas convaincu, mais il en fait néanmoins état dans son commentaire. 
place de sa sœur Eurynomé ${ }^{108}$. Cette dernière hypothèse semble la plus convaincante : l'autel serait bien celui des Charites et c'est près de la pierre qu'Asia serait honorée. La mythologie locale expliquait sans doute cette relation originale ${ }^{109}$. Si la restitution ö $\mu v v v \tau \imath$ de Herzog est correcte, c'est de ce verbe que dépend la proposition subordonnée introduite par ó $\pi \varepsilon i ̂$ et donc près d'Asia que le serment était prêté. Les qualités de courotrophes des Océanides sont bien attestées, dès la Théogonie d'Hésiode ${ }^{110}$.

Le relief classique de Mesaria laisse penser que les Charites de Cos étaient

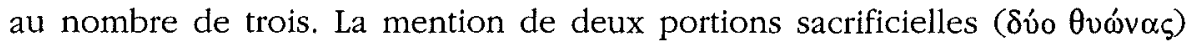
dans l'inscription a pourtant conduit Sokolowski à les réduire à deux. Pourrait-il s'agir d'une portion pour les Charites et d'une pour Asia ? Cela impliquerait que dès la déposition des Ovóvas sur l'autel des Charites, Asia leur était associée, mais que le dépôt d'entrailles (?) sur la pierre la concernait seule. De la même manière, la représentation de quatre femmes sur le relief hellénistique trouverait dans le rituel sa justification : ce ne seraient pas deux Charites et deux Nymphes, ou quatre Charites ${ }^{111}$, mais bien trois Charites et Asia.

Les témoignages sur l'éphébie sont presque inexistants dans l'île. Seule une série d'inscriptions en l'honneur de gymnasiarques d'éphèbes atteste l'existence de cette catégorie de jeunes gens à l'époque romaine ${ }^{112}$. Néanmoins, si l'on accepte de voir dans le rituel d'aspersion d'un autel et d'une pierre l'indice d'un rite de prestation de serment ${ }^{113}$, son insertion dans un calendrier sacrificiel implique la répétition annuelle de la cérémonic. Ce devait être la fréquence adoptée pour la prise en charge d'un groupe d'âge déterminé ${ }^{114}$. Le sanctuaire

108 LSCG, p. 261. Cf. supra, p. 197.

109 On ne connaît pas d'autre culte pour une Asia. PAUSANIAS (III, 24, 6-7) évoque une Athéna Asia à Las en Laconie et on a pu mettre cette épiclèse en relation avec la potnia a-si-wi-ja qui apparaît sur plusieurs tablettes en Linéaire B ( $c f$. G. MADDOLI, Potinija Asiwija, Asia e le relaztoni micenee con l'Anatolia settentrionale, in SMEA, 4 [1967], p. 11-22) et même aller jusqu'à établir une filiation directe entre le témoignage mycénien et l'inscription de Cos (Probabili ereditz̀ micenee nell'onomastica dei pyrgot a Teos e nel culto di Asia a COS, in SMEA, 7 [1968], p. 67-68).

110 HÉs., Théog., 346-348: Océan et Téthys enfantent une race de filles qui, avec Apollon et les

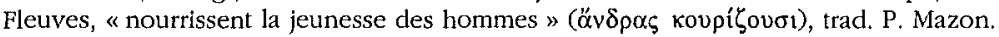

111 Cf. HARRISON, art. cit. (n. 59), n ${ }^{\circ} 42$,

112 W.R. PATON, E.L. HICKS, The Inscriptions of Cos, Oxford, 1891, n ${ }^{\circ}$ 106-111. Ces inscriptions ont été rééditées d'après les copies de Mario SEGRE, Iscrizioni di Cos, Roma, 1993 (Monografte della Scuola archeologica di Atene e delle missione ttaliane in Oriente, 6), $\mathrm{n}^{\circ}$ EV 94, 111, 17, 35 , 123,220 , auxquelles on peut ajouter EV 228, 249, 250a-b. - Une inscription funéraire métrique, peutêtre d'époque impériale, évoque la tristesse du mort de n'avoir pas vu sa fille sur le point de se

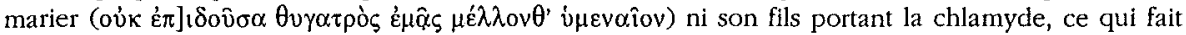

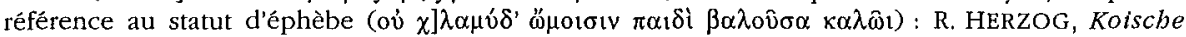
Forscbungen und Funde, Leipzig, 1899, p. 111-116, $\mathrm{n}^{\circ} 169,1.19-20$. Le thème est évidemment un topos dans ce type de circonstance (cf. HERzOG, ibid.).

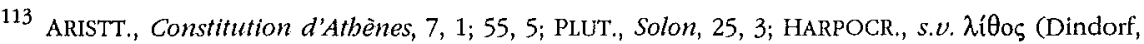
I [1853], p. 193); POLLUX, VIII, 86.

114 On ignore le moment exact de la prestation de serment des éphèbes athéniens. Est-ce lors de la cérémonie d'entrée (PÉLÉKIDIs, op. cit. [n. 44], p. 218-219) ou lors du passage au statut 
des Charites se situe à l'extérieur de la cité. On imagine mal des magistrats y prêter serment. Un tel cadre conviendrait bien, par contre, à des jeunes gens que le service éphébique, à l'instar des anciennes initiations juvéniles, cantonne autour de la cité ${ }^{115}$. Les cas d'Athènes et Sparte sont instructifs : de part et d'autre, les Charites accordent leur protection aux éphèbes.

Le relief dédié aux Charites par Peithanor aux alentour de 400, de facture attique $^{116}$, offre l'image d'un homme d'âge mûr et relève sans doute de la dévotion privée. Le relief hellénistique représente le dédicant dans une sorte de niche et Van Straten y a vu l'indice iconographique d'un ex-voto dédié à la suite d'un rêve ${ }^{117}$. E.B. Harrison interprète le personnage comme un enfant. Si les quatre femmes sont bien les Charites et Asia, l'« enfant » serait mis sous la protection des divinités du serment. La présence de Pan sur les deux reliefs atteste le caractère rural du sanctuaire, mais ne confirme ni n'infirme les hypothèses énoncées.

Un pas pourrait encore être franchi dans le parallèle avec la situation des éphèbes athéniens. Aux alentours de 200 avant notre ère, un certain Nicagoras,

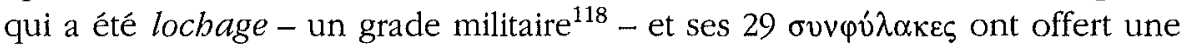
dédicace à Aphrodite 0 đók oo $^{119}$. La dédicace a été trouvée à Halasarna, un dème de la côté métidionale de l'île, où l'existence d'une garnison est attestée à l'époque hellénistique ${ }^{120}$. Le rapprochement avec le décret honorifique, émanant des soldats en garnison, trouvé à Rhamnonte dans le sanctuaire d'Aphrodite Hégémoné, est tentant.

Le culte de la déesse à Halasarna revêtait une importance telle que le prêtre d'Apollon devait obligatoirement lui offrir un sacrifice lors de différentes

d'hoplite (VIDAL-NAQUET, art. cit. [n. 45], p. 122-123, 146) ? Aucun argument ne permet d'emporter définitivement l'adhésion. - À Dréros, en Crète, une prestation de serment pour les jeunes gens est également attestée : ICret., I, IX, 1. Cf. M. BILE, Les termes relatifs à l'initiation dans les inscriptions crétolses (VIIe-Ier siècles av. J.-C.), in A. MOREAU (éd.), L'initiation, I : Les rites d'adolescence et les mystères (Montpellier; 11-14 auril 1991), Montpellier, 1992, p. 11-17, surtout p. 14-15.

115 Sur ce point, VIDAL-NAQUET, art. cit. (n. 45), p. 121-122. - La mention des oliviers au milieu desquels s'élèvent la pierre associée au sacrifice implique néanmoins un endroit cultivé, mais HERZOG (op. cit. [n. 99], p. 13) a bien mis en évidence, exemples à l'appui, la récurrence des oliviers en relation avec ce type de serment.

116 KONSTANTINOPOULOS, art. cit. (n. 94).

117 F. VAN STRATEN, Daikrates' Dream. A votive rellef from Kos, and some otber kat'onar dedications, in BABesch, 51 (1976), p. 1-38.

118 On connaît, à Athènes, des lochages de l'armée (ARISTT., Const. Atb., 61, 3) et des lochageséphèbes, un grade à l'intérieur du corps des éphèbes (PÉLÉKIDIS, op. cit. [n. 44], p. 109-110: « je me demande si ces lochages ne servaient pas comme officiers des soldats mercenaires qui formaient des patrouilles avec les éphèbes et gardaient les forts de l'Attique »).

119 A. MAIURI, Nuova silloge epigrafica di Rodi e Cos, Firenze, 1925, no 675.

120 SHERWIN-WHITE, op. cit. (n. 97), p. 171-172. - Le serment de Dréros (supra, n. 114) évoque la présence des jeunes en garnison (1. 126-127; cf. H. VAN EFFENTERRE, Fortins crétois, in Mélanges Cb. Picard, II, Paris, 1949, p. 1033-1034). 
fêtes $^{121}$. Dans le dème d'Isthmos, Aphrodite Pandamos recevait le sacrifice d'un chevreau selon un calendrier cultuel du IIIe siècle av. J.-C. ${ }^{122}$ et l'hypothèse d'une relation entre l'épiclèse de la déesse et le synocisme de 366 est plausible ${ }^{123}$. Le même calendrier sacrificiel atteste l'existence d'un culte d'Homonoia ${ }^{124}$ qui pourrait avoir été établi ou revitalisé au sortir d'une situation de crise ${ }^{125}$.

Les Charites, Aphrodite Pandémos, Homonoia, autant de divinités qui évoquent l'instauration du culte du Démos et des Charites à Athènes à la même époque, et les honneurs rendus à une Aphrodite «conductrice du peuple ». Si les éphèbes de l'île de Cos tenaient garnison aux endroits stratégiques comme leurs homologues athéniens, peut-être la dédicace d'Halasarna à une Aphrodite «attentive » évoque-t-elle les qualités de courotrophe de la déesse, partagées par les Charites aux belles boucles que le plus ancien des deux reliefs de Cos montre dansant autour de l'autel de leur téménos.

Vinciane PIRENNE-DELFORGE Cbercheur qualifié du F.N.R.S.

Université de Liège

Centre d'Histoire des Religions

Place du 20-Août, 32

B - 4000 LIÈGE

121 LSCG, n 172. Ce règlement, qui date du IIle siècle av. J.-C., évoque des sacrifices de chèvres pour la déesse; il mentionne aussi un jardin sacré et un bain.

$122 L S C G, \mathrm{n}^{\circ} 169$ A, 1. 12-13.

123 Pour le cas athénien, $c f$. PIRENNE-DELFORGE, op. cit. (n. 38), p. 448-450. Je nuance ici l'affirmation de la p. 450 selon laquelle les Charites étaient des divinités de la végétation!

124 LSCG, $\mathrm{n}^{\circ} 169,1.4$. - L'inscription EV 18 de SEGRE (op. cit. [n. 112]) est une dédicace à Aphrodite et à Homonoia de la part de magistrats religieux.

125 SHERWIN-WHITE, op. cit. (n. 97), p. 330. 